\title{
Study on migration and change of petroleum pollutants in soil and pollution evaluation with data mining technology
}

\author{
Gao Lijuan ${ }^{1}$ \\ ${ }^{1}$ China's Yunnan Nuclear Industry, Kunming, Yunnan, 650000
}

\begin{abstract}
To study the migration and variation rule of petroleum pollutants in soil and evaluate the soil condition, the field investigation is carried out in a refinery in Yunnan Province and the relevant data are collected. Then, soil sampling and leaching experiments are carried out on the land in the refinery. Finally, the experimental data are sorted out. The experimental results show that the pollution of surface soil in refinery is the most serious. Because of the adsorb-ability of the soil, the content of petroleum pollutants in the deep soil is less. The results of leaching experiments show that the diffusion process of petroleum pollutants is greatly affected by random factors in horizontal diffusion. In the vertical direction, the depth of soil will reduce the diffusion speed of petroleum pollutants. This exploration provides a reference for the study of the migration and variation rule of petroleum pollutants in soil.
\end{abstract}

\section{Introduction}

Soil pollution in China mainly comes from the discharge of industrial waste. Effective control plans should be made for main pollution problems in each region for the purpose of soil pollution prevention. With the rapid development of the oil industry in China, pollution has occurred in the process of oil exploitation, refining and transportation. Oil volatilization and leakage and the discharge of oily sewage in the process will cause ecological pollution to the atmosphere, water, soil and other environmental media. Oil pollutants entering the soil will destroy the original soil structure and property and reduce soil permeability. Meanwhile, the high polymer petroleum hydrocarbons remaining in the soil will form a mucous membrane on the root of plants and affect the respiration and nutrient absorption of the root, resulting in the death of vegetation. Moreover, soil pollution is concealed. The enrichment of oil pollution makes it difficult to be found ${ }^{[1]}$.

Harmsen and Rietra (2018) studied the biodegradation of polycyclic aromatic hydrocarbons (PAHs) and total petroleum hydrocarbons (TPHs) in sediments and soil over a 25 -year period. The experimental results showed that polycyclic aromatic hydrocarbons degraded rapidly in the first year, at a slower rate during the subsequent 6 years and very slowly after 7 years, and that the aromatic ring was not degraded until 25 years later. However, the total petroleum hydrocarbons in the soil can hardly be degraded [2]. Pabón et al. (2019) studied the environmental pollution in the process of oil refining and transportation and detected and characterized the pollution status of petroleum hydrocarbons with diffuse reflection and imaging (hyperspectral) spectroscopy. The experimental results showed that the technology could effectively identify petroleum hydrocarbon leakage and contaminated soil, thereby reducing environmental pollution caused by petroleum hydrocarbon leakage effectively ${ }^{[3]}$.

An oil refinery in Yunnan Province was selected in this paper through field investigation for study on the law of the migration and change of oil pollutants in the soil. First, samples were collected from the soil in the oil refinery and bagged and marked. Second, the soil collected was pretreated, and the migration process of oil pollutants in the soil was studied through leaching experiments. Finally, the experimental data obtained was analyzed and summarized and the soil pollution of the refinery was evaluated.

\section{Study on the law of migration and change of oil pollutants}

Oil compounds, industrial pollutants with great harm and a long cycle of danger, will migrate to the surrounding environment in different forms under certain conditions, causing secondary pollution. Oil, a complex mixture composed of various hydrocarbons, can be divided into alkanes, cycloalkanes, alkenes and aromatic hydrocarbons by the structure of hydrocarbons. Among them, PAHs are relatively inert compounds that can exist in the environment for a long time. Some PAHs are highly toxic and can accumulate in the human body through the food chain, which is an important cause of human cancer. PAHs in the atmosphere and soil are in an adsorbed state as they are insoluble in water and easily adhere to solid particles. We can master the tendency of pollutants to enter the environment, thereby 
strengthening and controlling the process to reduce the resulting pollution hazard, by studying and grasping the law of the migration and change of oil pollutants in soil and water ${ }^{[4]}$.

Soil is a special porous medium with porosity and compressibility. Therefore, the migration and diffusion process of PAHs in soil is similar to that in porous media, including physical, chemical and biological processes resulting in changes in their temporal and spatial distribution in soil. The physical process includes adsorption and dispersion, etc., and the chemical process includes precipitation, dissolution and redox reactions, etc. The biological process includes biodegradation and related biochemical reactions, etc. The three processes interact and correlate with each other, causing PAHs to migrate and diffuse in the soil. Adsorption and desorption play a vital role in the migration process of PAHs in the environment. Meanwhile, the degree of adsorption and desorption of PAHs directly affects their mobility, volatility and biodegradability in the environment and their toxic effect on organisms ${ }^{[5]}$.

The adsorption process of organic matters in the soil involves two patterns. First, a similar process of dissolution of organic pollutants in a water-containing soil medium, which is only related to the solubility of organic matters. Second, the surface adsorption of soil minerals, i.e. the adsorption of organic pollutants through the action of various chemical bonds, in which process a lot of heat is released ${ }^{[6]}$. The adsorption equilibrium process of organic matters can be studied based on the adsorption/desorption model. The equation for the Langmuir isothermal equilibrium adsorption model is:

$C_{e}$ refers to the equilibrium concentration of solutions in the environment of the attachment. $q_{e}$ refers to the concentration of the attachment in the absorbate at equilibrium. $\alpha$ is the Langmuir constant at a certain temperature. $Q$ is the upper limit of the adsorption potential at a certain temperature.

In the production process of the refinery, some oil pollutants enter the ditch and seepage pit or drip onto the ground, forming pollution sources with a certain area, and spread to the surrounding soil during the transportation and treatment of waste water, sewage and crude oil pipelines. After the oil pollutants enter the soil, those adsorbed in the soil can enter the water under certain conditions, resulting in damage to the groundwater quality. Methods for the study on the diffusion of oil pollutants in the soil include the indoor soil column leaching experiment, field experiment and numerical simulation method ${ }^{[7]}$.

Following field investigation of the refinery, the waste water pool, sewage outlet and pipe opening in the refinery were selected for study. The change law of pollutant migration was obtained through collection and analysis of contaminated soil samples in the refinery. First, a pit, $2 \mathrm{~m}$ long, $1 \mathrm{~m}$ wide and $1 \mathrm{~m}$ deep, was excavated along the soil profile with the profile facing the sunlight for the convenience of later observation and photography. Then, the morphological characteristics of the soil profile were observed and described. Started with the soil surface, soil samples of $500 \mathrm{~g}$ were taken for every $20 \mathrm{~cm}$ layer. The soil in contact with the shovel was removed with a bamboo knife and bagged and marked in the process of collection. The soil samples collected were dried in a cool and ventilated environment, had the debris removed and were then ground before kept in dark places. The data acquired after processing is shown in Tables 1-3.

Table 1 Soil contamination at pipe crossings

\begin{tabular}{|c|c|c|c|c|c|}
\hline $\begin{array}{c}\text { Soil depth/ } \\
\mathrm{cm}\end{array}$ & $\begin{array}{c}\begin{array}{c}\text { Source } \\
\text { distance/ m }\end{array} \\
\begin{array}{c}\text { Petroleum } \\
\text { content } /(\mathrm{mg} / \mathrm{kg})\end{array} \\
\end{array}$ & 5 & 10 & 25 & 50 \\
\hline \multicolumn{2}{|r|}{10} & 70 & 48 & 35 & 25.3 \\
\hline \multicolumn{2}{|r|}{30} & 41.8 & 35.5 & 27.3 & 23 \\
\hline \multicolumn{2}{|r|}{50} & 32 & 28 & 25 & 18.1 \\
\hline \multicolumn{2}{|r|}{70} & 25 & 23 & 20 & 12.2 \\
\hline
\end{tabular}

Table 2 Soil Contamination in Sewage Pool

\begin{tabular}{|c|c|c|c|c|c|}
\hline \multirow{2}{*}{$\begin{array}{c}\text { Soil depth/ } \\
\mathrm{cm}\end{array}$} & $\begin{array}{c}\text { Source distance/ m } \\
\text { Petroleum } \\
\text { content/(mg/kg) }\end{array}$ & 5 & 10 & 25 & 50 \\
\hline 10 & 37 & 25.5 & 25 & 23 \\
\hline 30 & 25.3 & 24.6 & 18 & 16.8 \\
\hline 50 & 23.5 & 20 & 12.8 & 11 \\
\hline 70 & 15 & 14.1 & 10 & 8 \\
\hline
\end{tabular}


Table 3 Soil contamination at sewage outlets

\begin{tabular}{|c|c|c|c|c|c|}
\hline \multirow{2}{*}{$\begin{array}{c}\text { Soil depth/ } \\
\mathrm{cm}\end{array}$} & $\begin{array}{c}\text { Source distance/ } \\
\mathrm{mm}\end{array}$ & 5 & 10 & 25 & 50 \\
$\mathrm{content/( \textrm {mg } / \mathrm { kg } )}$ & 5 & 49.3 & 49.3 & 37.5 & 23 \\
\hline 10 & 42.3 & 37.6 & 24.8 & 25.3 \\
\hline 30 & 20 & 12.3 & 10.6 & 9 \\
\hline 50 & 10 & 10 & 5 & 3 \\
\hline 70 & & & & \\
\hline
\end{tabular}

This paper studies the vertical and horizontal migration and change process of oil pollutants in the soil through soil column leaching experiments.

(1) Horizontal leaching experiment. The uncontaminated soil collected from the producing wellhead was loaded into the experimental container, a long trough made of organic glass, in its natural order and with its natural volume weight maintained. One end of the long trough was covered with a layer of oil sludge and sand. Deionized water was used as the leachate for regular water replenishment. The oil content of the exudate from the bottom of the soil column was measured. The oil content in the soil was analyzed after the 60-day experiment was completed.
(2) Vertical leaching experiment. The uncontaminated soil collected was loaded into a vertical cylinder in the natural order and with its natural volume weight maintained. A $1 \mathrm{~m}$ high soil column was made, the upper end of which was covered with a layer of oil sludge and sand. Deionized water was used as the leachate for regular water replenishment. The exudate from the bottom was analyzed. The experiment lasted for 60 days.

The oil sample prepared was tested based on the national standard Water Quality - Determination of Petroleum, Animal Fats and Vegetable Oils - Infrared Spectrophotometry.

\section{Results and discussions}

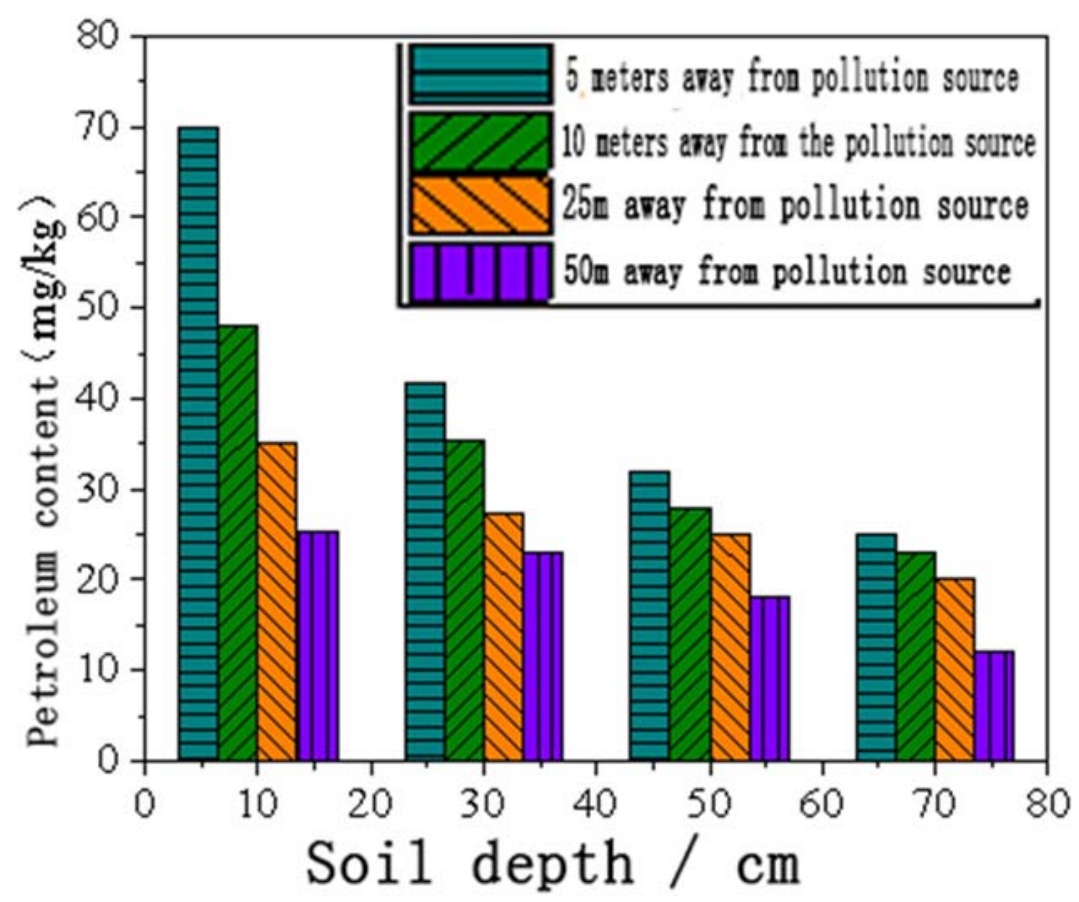

Figure 1 Soil pollution at pipe mouth. 


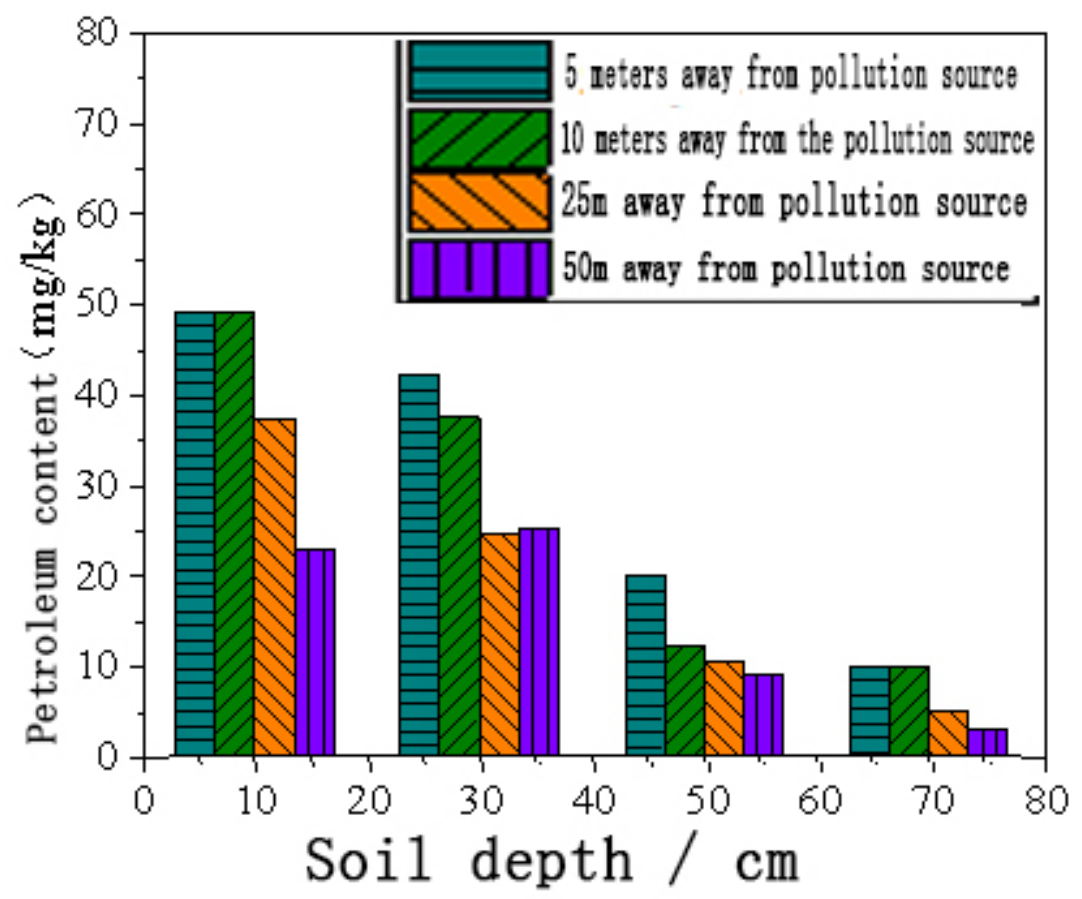

Figure 2 Soil pollution in sewage ponds.

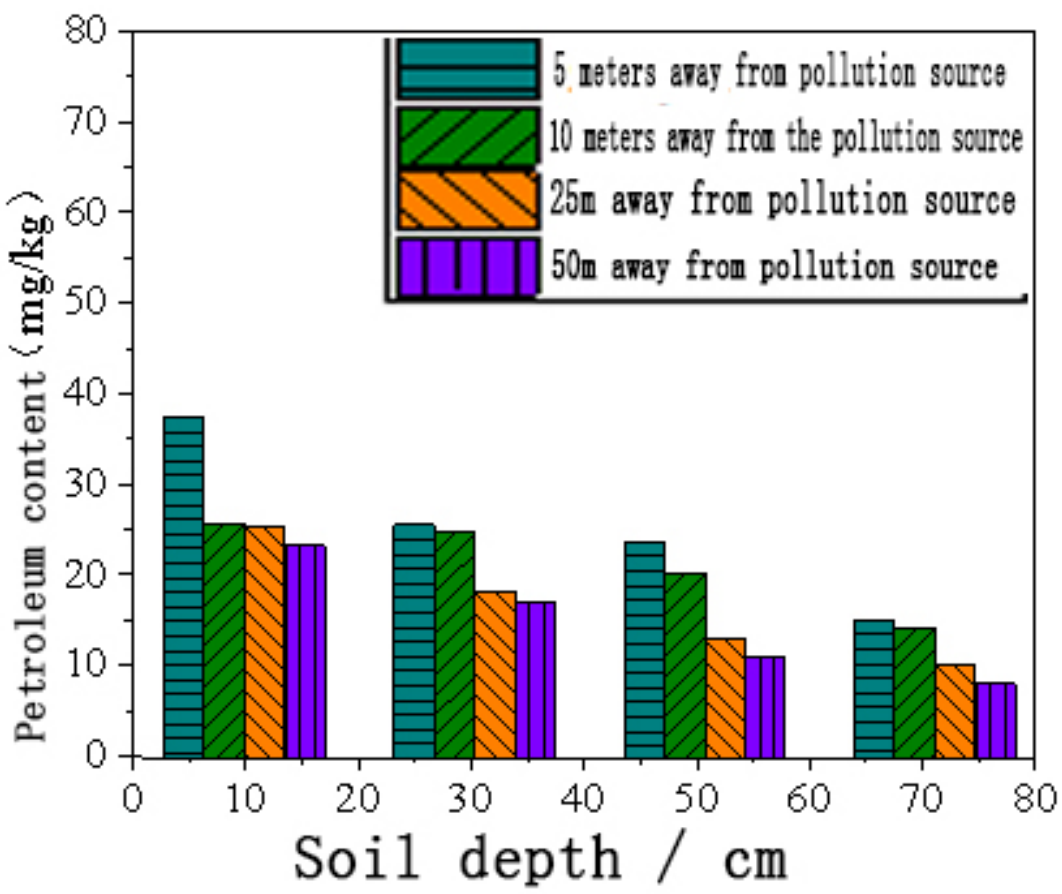

Figure 3 Soil pollution at sewage outlet.

Figures 1 to 3 show the test results of the soil from the pipe opening, waste water pool and sewage outlet of the refinery, according to which the surface soil in the refinery is most severely polluted and the content of oil pollutants decreases gradually with the increase of soil depth. The farther away from the pollution sources, the lower the content of oil pollutants in the soil. Crude oil pollutants produced fall to the surface of the soil during oil production. The content of oil pollutants in deep and distant soil decreases due to the low oil permeability and the adsorption of the soil.

The results of the horizontal soil leaching experiment are shown in Figure 4. 


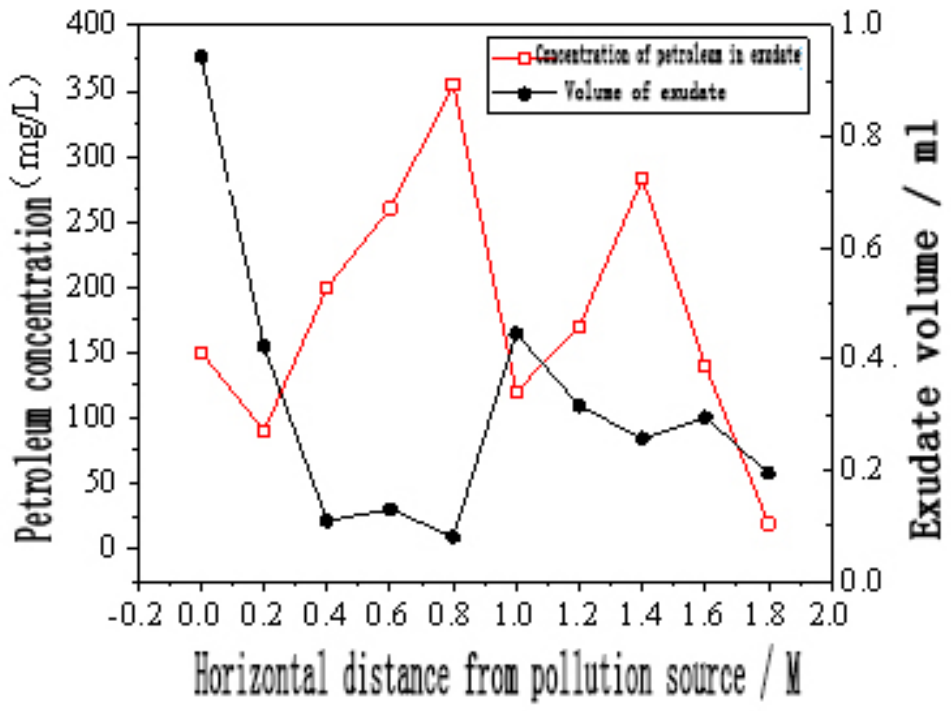

Figure4 Experimental results of horizontal leaching

According to Figure 4, the leaching results fluctuate greatly and the oil content in the exudate does not show an obvious law possibly due to the greater influence of random factors on the horizontal migration of oil pollutants.

The results of the vertical soil leaching experiment are shown in Figure 5.

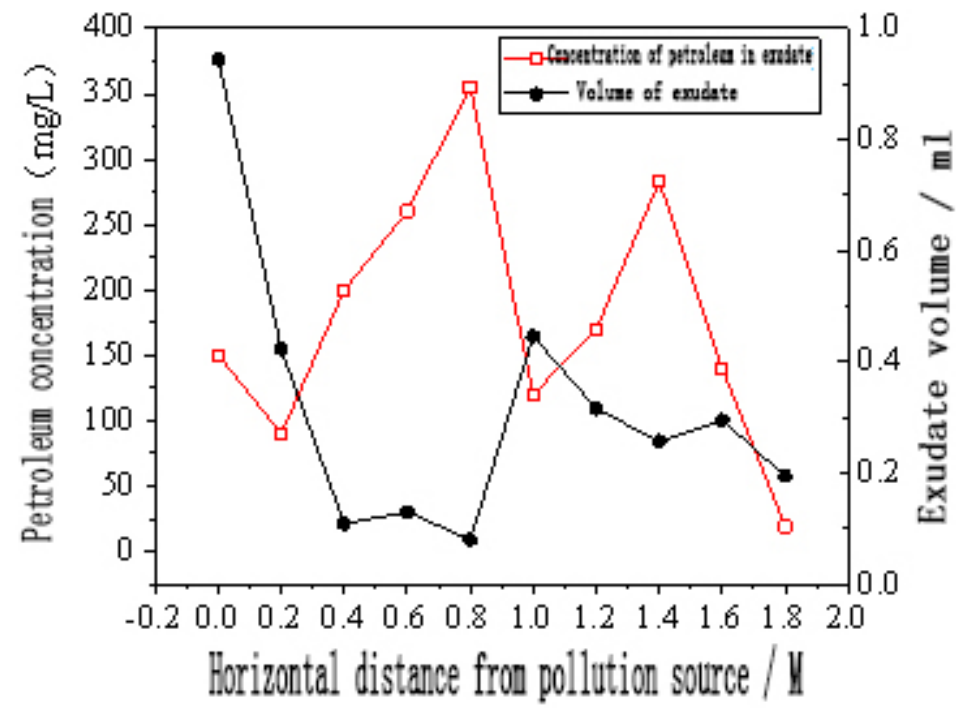

Figure 5 Experimental results of vertical leaching.

It can be seen from Figure 5 that the time of exudation gradually delays as the soil depth increases. The concentration of oil pollutants in the soil exudate gradually decreased with the progress of the experiment. It tended to decrease slowly after 10 days of leaching and was thereafter reduced and maintained at about 0.25 $\mathrm{mg} / \mathrm{L}^{-1}$.

According to vertical and horizontal soil leaching experiments, the time of exudation delays as the soil depth increases in the vertical direction. As the experiment progressed, the concentration of the exudate decreased gradually and its change tended to be gentle after 10 days. In the horizontal direction, oil pollutants are greatly affected by random factors. The concentration of oil pollutants in the soil shows a downward trend with the increase of the distance from pollution sources. Therefore, soil can highly intercept and adsorb oil pollutants.

\section{Conclusion}

To study the law of the migration and change of oil pollutants in the soil, this paper conducted field investigation on the environmental pollution of soil in an oil refinery in Yunnan and collected the data. Finally, the soil near three pollution sources in the refinery was selected for study. First, soil samples were collected and analyzed. Then, the law of the migration and change of 
oil pollutants in the soil was obtained through leaching experiments. The results show that the soil near the pollution sources is most severely polluted and that, the farther away from the pollution source, the lower the content of oil pollutants in the soil. The content of oil pollutants decreases as the soil depth increases due to the adsorption of the soil. This paper has some shortcomings, including the inadequate number of test samples selected. Meanwhile, the adsorption of different pollutants in the soil is not the same. Therefore, it is necessary to test different pollutants in the soil by category in subsequent experiments.

\section{References}

1. Li Jia, Cao Xingtao, Sui Hong, et al. Current situation and prospect of petroleum contaminated soil remediation technology. Journal of Petroleum (Petroleum Processing),2017,33(5), pp.811-833.

2. Harmsen J,Rietra R P J J.25years monitoring of PAHs and petroleum hydrocarbons biodegradation in soil.Chemosphere,2018,207,pp.229-238.

3. Pabon R E C,de Souza Filho C R,de Oliveira W J.Reflectance and imaging spectroscopy applied to detection of petroleum hydrocarbon pollution in bare soils.Science of the total environment,2019,649,pp.1224-1236.

4. Qian Cheng, Mu Wenping, Xing Yuan, et al. Numerical simulation of petroleum pollutant migration in a gas field. Environmental Engineering ,2016,34(4), pp.68-72.

5. Zhang Shudong, Weng Chen, Huang Huiqin, et al. Seasonal variation of soil petroleum pollutants in Xisha wetland of Chongming and its influence on vegetation types. Ecoenvironmental Journal ,2016,25(2), pp.300-306.

6. Liu Yulong, Wu Weiyang, Fan Junxin, et al. Experimental study on anti-seepage performance of oil pollutants in Yunnan compacted clay layer. Geoscience frontiers ,2019,26(4), pp.273-278.

7. Wang Baoshan, Wen Chengcheng, Sun Qinchuan, et al. Effects of petroleum hydrocarbon pollution on soil microbial diversity in frozen area of Beiluhe region of Qinghai-Tibet Plateau. Journal of Environmental Engineering ,2018,12(10), pp.29172928. 